Received: 2016.08.15

Accepted: 2016.09 .08

Published: 2017.04 .19
Authors' Contribution:

Study Design A

Data Collection B

Statistical Analysis C

Data Interpretation D

Manuscript Preparation E

Literature Search F

Funds Collection G

Corresponding Author:

Source of support:

\title{
Insulin Growth Factor 1 Protects Neural Stem Cells Against Apoptosis Induced by Hypoxia Through Akt/Mitogen-Activated Protein Kinase/Extracellular Signal-Regulated Kinase (Akt/MAPK/ERK) Pathway in Hypoxia-Ishchemic Encephalopathy
}

BCEF Bing Zhao

ADEF Zebao Zheng
Department of Children Rehabilitation, Jining No. 1 People's Hospital, Jining, Shandong, P.R. China

Background: Insulin growth factor 1 (IGF-1) is reported to modulate cell growth and acts as potential therapy for traumatic brain injury. This study was designed to investigate the effect of IGF-1 on hypoxia-induced apoptosis in neural stem cells (NSCs).

Material/Methods: A hypoxia model was constructed using NSCs separated from the hippocampus of rat. NSCs were divided into four groups: cells under normoxic conditions that acted as controls (C group), cells under hypoxia (H group), cells under hypoxia with IGF-1 (HI group), and cells under hypoxia with IGF-1 as well as picropodophyllin (PPP), which acts as an inhibitor of the IGF-1 receptor (HIP group). The cell viability and apoptosis were respectively measured by 3-(4,5-dimethylthiazol-2-yl)-2, 5-diphenyltetrazolium bromide (MTT) assay and flow cytometry. Finally, the phosphorylation levels of apoptosis-associated proteins and key kinases in the phosphatidylinositol3-kinase (PI3K)/AKT and the mitogen-activated protein kinase/extracellular signal-regulated kinase (MAPK/ERK) pathways were detected by Western blot analysis.

Results: In comparison with the $\mathrm{H}$ group, the cell viability was increased while the cell apoptosis was reduced by IGF1 in the $\mathrm{HI}$ group. Besides, the expression levels of Bax, cytochrome $c$, and activated caspase- 3 were all improved in the $\mathrm{H}$ group, and the remarkable differences were eliminated in the $\mathrm{HI}$ group compared with the $\mathrm{C}$ group. The expression level of Bcl-2 was the opposite. Additionally, down-regulations of phosphorylated AKT, MAPK, and ERK induced by hypoxia were all improved by IGF-1. All the influences of IGF-1 were weakened by addition of PPP.

Conclusions: $\quad$ IGF-1 increased cell viability while decreasing apoptosis in hypoxic NSCs through the PI3K/AKT and MAPK/ERK pathways.

MeSH Keywords: $\quad$ Apoptosis • Cell Survival • Insulin-Like Growth Factor Binding Protein $1 \bullet$ MAP Kinases • Neural Stem Cells • Phosphatidylinositol 3-Kinases

Full-text PDF: http://www.medscimonit.com/abstract/index/idArt/901055

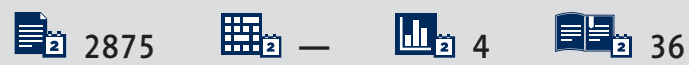




\section{Background}

Perinatal hypoxic-ischemic encephalopathy (HIE) is an acquired brain injury that mainly happens in newborn infants $[1,2]$. The severity, duration, and timing of the HIE antenatal injury are difficult to discern [3]. In live neonates, the incidence of HIE ranged from $0.1 \%$ to $0.8 \%$ in developed countries and even as high as $2.6 \%$ in underdeveloped countries [4]. In spite of widespread use of therapeutic hypothermia, $40 \%$ of neonates with HIE have neurological disability at 18-24 months of age [5].

Although the brain is $2 \%$ of the total body weight, it consumes $20 \%$ of the total body oxygen, which means that the brain has a relatively high rate of oxygen consumption [6]. In addition to the limited oxygen reserves in the brain, the central nervous system (CNS) is highly susceptible to anoxia and displays low tolerance to hypoxia [7]. Neural stem cells (NSCs) are the cells exhibiting stem cell properties that possess the ability to self-renew and to generate major cell types in the CNS $[8,9]$. Hypoxia disrupts the homeostasis of the microenvironment and plays a role in cell proliferation and differentiation. There are various overwhelming evidences confirming that a low oxygen level facilitates the survival of myoblasts [10], and hematopoietic [11], neural [12], and human embryonic stem cells [13]. However, long-term anoxia is considered to suppress cell growth of epithelial stem cells [14].

Insulin growth factor 1 (IGF-1) consists of 70 amino acid residues accompanied by three disulfide bridges [15]. In particular, IGF-1 is one of the neurotrophins that modulate cell growth [16]. IGF-1 has been proven to bind with the IGF-1 receptor (IGF-1R) and then activate the phosphatidylinositol3-kinase (PI3K)/AKT signaling pathway, thereby facilitating cell proliferation and differentiation [17]. Recent studies illustrated that IGF-1 influenced early embryonic development via increasing the number of cells [18]. Moreover, IGF-1 is reported to be an essential growth factor for promoting proliferation and suppressing apoptosis of neural progenitor-like cells [19]. Rubovitch et al. also suggested that IGF-1 was a potential neuroprotective therapy for traumatic brain injury-induced damage [20]. Picropodophyllin (PPP) is a selective inhibitor of tyrosine phosphorylation of IGF-1R [21] and could effectively suppress the activation of IGF-1R [22]. To our knowledge, the modulating effect of IGF-1 on hypoxic NSCs has seldom been investigated. Hence, the aim of our research was to explore the effect of IGF-1 with or without addition of PPP on NSC apoptosis induced by hypoxia. The mechanism involved was also studied.

\section{Material and Methods}

\section{Experimental animals}

Pregnant Sprague-Dawley (SD) rats were purchased from the experimental animal center of Nantong University (Jiangsu, China). Every animal study was conducted in accordance with the United States National Institutes of Health Guide for the Care and Use of Laboratory Animals. The experimental protocols were approved by our local ethics committee. All efforts were made to minimize the number and suffering of rats involved in our study.

\section{Cell culture}

NSCs from the hippocampus were obtained as described previously [23]. Briefly, following anesthesia with chloral hydrate ( $2 \mathrm{~mL} / \mathrm{kg}$ of body weight), the hippocampus was isolated from 14-day SD rat embryos and placed on ice. After digestion of the hippocampus with $0.125 \%$ trypsin (Gibco, Grand Island, New York, USA), the NSCs were dissociated into single-cell suspensions. The cell suspensions with a density of $1 \times 10^{4}$ cells/ $\mathrm{mL}$ were cultured in 1: 1 Dulbecco's modified Eagle's medium (DMEM)/F12 (Gibco, Carlsbad, California, USA) containing 2\% B27 (Invitrogen, Carlsbad, California), basic fibroblast growth factor (bFGF) 10 ng/mL (Peprotech, Rocky Hill, New Jersey), epidermal growth factor (EGF) $10 \mathrm{ng} / \mathrm{mL}$ (Peprotech, Rocky Hill, $\mathrm{NJ}$ ), and penicillin/streptomycin $100 \mathrm{U} / \mathrm{mL}$ (Beyotime, Shanghai, China). The cultures were maintained in a humidified incubator at $37^{\circ} \mathrm{C}$, with $95 \%$ air and $5 \% \mathrm{CO}_{2}(\mathrm{v} / \mathrm{v})$. Five days later, neurospheres were centrifuged, resuspended, and plated on 96-well plates at 1-2 cells/well. Then, the digestion and passage were conducted again as before. In order to fetch neurospheres originating from a single primary cell, cells were passaged three times. Digested from the neurospheres, the NSCs used for subsequent experimentations were maintained under the same culturing condition with neurospheres.

\section{Construction of hypoxia model}

The hypoxia environment was made by placing NSCs in a humidified chamber (StemCell Technologies, Vancouver, Canada) with a gas mixture of $\mathrm{N}_{2}, \mathrm{CO}_{2}$, and $\mathrm{O}_{2}$ at $37^{\circ} \mathrm{C}$. The percentage of the three gases was $94 \%, 5 \%$, and $1 \%$, respectively. In order to ascertain the most suitable duration of hypoxia for further experiments, the cells were transferred from the normoxic incubator to the hypoxic incubator for different durations $(0,2$, $4,6,8$, and $10 \mathrm{~h}$ ). The control group was cultured in normoxic conditions all the time for the same durations.

\section{IGF-1 and PPP treatments}

NSCs were hypoxia cultured with IGF-1 at various concentrations $(0,10,50,100$, or $200 \mathrm{ng} / \mathrm{mL})$. After that, the cell viability 
of NSCs induced with different concentrations of IGF-1 was assayed. The group without IGF-1 induction acted as the control.

NSCs for further studies were divided into four groups, namely, the $\mathrm{C}, \mathrm{H}, \mathrm{HI}$, and HIP groups. Cells in the $\mathrm{C}$ group were incubated without any treatment, cells in the $\mathrm{H}$ group were incubated with hypoxia treatment, cells in the $\mathrm{HI}$ group were incubated with hypoxia treatment accompanied by IGF-1, and cells in the HIP group were incubated with hypoxia treatment accompanied by IGF-1 and PPP $500 \mathrm{nmol} / \mathrm{L}$.

\section{Cell viability assay}

The cell viability after different treatments was measured by 3-(4,5-dimethylthiazol-2-yl)-2,5-diphenyltetrazolium bromide (MTT) colorimetric assay according to the standard method described before [24]. In brief, $20 \mu \mathrm{L}$ of MTT ( $5 \mathrm{mg} / \mathrm{mL}$ ) was added to each well of 96-well plates. After $4 \mathrm{~h}$ of incubation at $37^{\circ} \mathrm{C}, 200 \mu \mathrm{L}$ of dimethyl sulfoxide (DMSO) (Sigma, St Louis, Missouri, USA) was added to terminate the reaction. At last, the plates were shaken vigorously for $10 \mathrm{~min}$, and the levels of reduced MTT were measured by a plate reader (Biotek Synergy 2, Winooski, Vermont, USA) at a wavelength of $570 \mathrm{~nm}$.

\section{Cell apoptosis assay}

The quantitative assessment of NSC apoptosis was conducted by flow cytometry using the Annexin V-FITC/propidium iodide (PI) apoptosis detection kit (Beijing Biosea Biotechnology, Beijing, China). In brief, the cells were resuspended with a density of $1 \times 10^{5}$ cells/well in binding buffer after twice washing with cold phosphate-buffered saline (PBS). Then, both the adherent and floating cells were treated with $10 \mu \mathrm{L}$ of Annexin V-FITC and 5 $\mu \mathrm{L}$ of $\mathrm{PI}$ according to the manufacturer's instruction. Finally, the cells were measured with a flow cytometer (Beckman Coulter, USA), which distinguished apoptotic cells (Annexin-V positive and PI-negative) from necrotic cells (Annexin-V and PI-positive).

\section{Western blot analysis}

Proteins in NSCs were extracted using RIPA lysis buffer (Beyotime Biotechnology, Shanghai, China) and protease inhibitors (Roche, Indianapolis, Indiana, USA) according to the manufacturer's instruction. The protein contents were quantified using the BCA $^{\mathrm{Tm}}$ Protein Assay Kit (Pierce, Appleton, Wisconsin, USA). Equivalent amounts of proteins were loaded on $12 \%$ polyacrylamide gels and separated through sodium dodecyl sulfate-poly-acrylamide gel electrophoresis (SDS-PAGE). Afterwards, the proteins on the gels were transferred to polyvinylidene difluoride (PVDF) membranes (Millipore, Billerica, Massachusetts, USA). The membranes carrying proteins were incubated for $1 \mathrm{~h}$ with blocking buffer that contained $0.1 \%$ Tween 20, $20 \mathrm{mM}$ Tris-HC, and $137 \mathrm{mM} \mathrm{NaCl}$ (TBST) and 4\% bovine serum albumin (BSA; Sigma-Aldrich, Sydney, NSW, Australia) at room temperature. After three rounds of rinsing with TBST, the membranes were incubated overnight at $4^{\circ} \mathrm{C}$ with primary antibodies against mammalian B cell lymphoma-2 (Bcl-2, 15071), Bcl-2-associated X protein (Bax, 14796), cytochrome c (11940), cleaved capase-3 (9661), pro capase-3 (9662), GAPDH (2118), phosphorylated mitogen-activated protein kinase ( $p$-MAPK, 4370), MAPK (4695) (all from Cell Signaling Technology, Danvers, Massachusetts, USA), phosphorylated AKT (p-AKT, sc-101629), AKT (sc-8312), phosphorylated extracellular signal-regulated kinase (p-ERK, sc-16981-R), and ERK (sc-292838) (all from Santa Cruz Biotechnology, California, USA). Membranes were then incubated with second antibodies at room temperature for 2 h. After rinsing with TBST for three times, the membranes carrying blots and antibodies were transferred into the Bio-Rad ChemiDoc $^{\mathrm{TM}}$ XRS system with addition of $200 \mu \mathrm{L}$ of Immobilon Western Chemiluminescent HRP Substrate (Millipore). The signals were captured and the intensity of the bands was quantified using Image Lab ${ }^{\text {TM }}$ Software (Bio-Rad, Shanghai, China). GAPDH was used as an internal control.

\section{Statistical analysis}

All results were collected from three independent experiments. Data from multiple experiments are presented as the mean \pm standard deviation (SD). Statistical analyses were performed using GraphPad Prism 5.0 software (GraphPad, San Diego, California, USA). The $P$ values for multiple comparisons were calculated by one-way analysis of variance (ANOVA). A $P$ value of $<0.05$ was considered statistically significant.

\section{Results}

\section{Construction of hypoxia model}

To construct the hypoxia model of NSCs, the most suitable duration of hypoxia was evaluated by MTT assay. Figure 1 shows that when the hypoxia time lasted for $2 \mathrm{~h}$, the cell viability was accelerated compared with control $(F(5,12)=94.78$, $P<0.01)$. With the extension of hypoxia time, the cell viability was decreased significantly in comparison with control $(\mathrm{F}(5,12)=94.78, P<0.05$ or $P<0.001)$. Cell viability at $6 \mathrm{~h}$ was markedly decreased in comparison with that of cells at $4 \mathrm{~h}$ $(F(5,12)=94.78, P<0.01)$. However, the difference in cell viability between $6 \mathrm{~h}$ and $8 \mathrm{~h}$, as well as that between $8 \mathrm{~h}$ and $10 \mathrm{~h}$, was not significant. Thus, the hypoxia model was constructed with exposure to hypoxia for $6 \mathrm{~h}$.

\section{IGF-1 enhanced cell viability of hypoxic NSCs}

The most suitable concentration of IGF-1 induction for hypoxic NSCs was determined by MTT assay. When the concentration of 


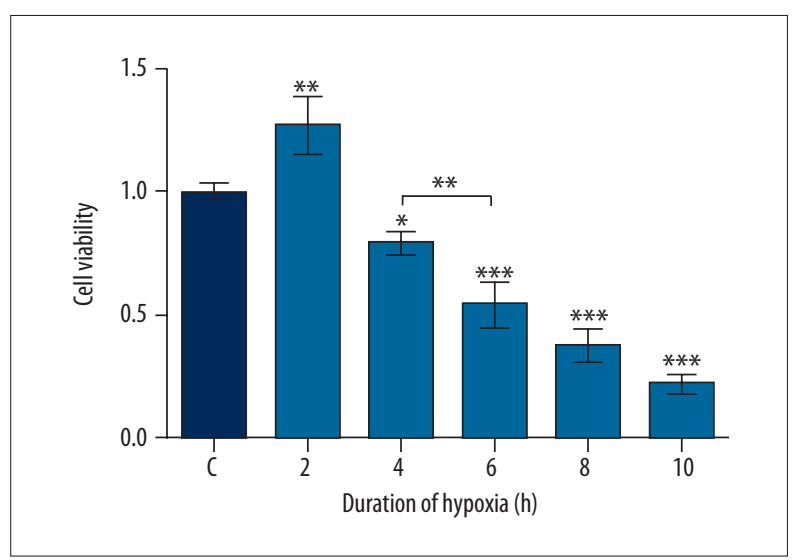

Figure 1. Effect of hypoxic duration on cell viability. Cell viability was measured by 3-(4,5-dimethylthiazol-2-yl)-2, 5-diphenyltetrazolium bromide (MTT) assay. Data presented are the mean \pm SD of three independent experiments. ${ }^{*} P<0.05$; ${ }^{* *} P<0.01$; ${ }^{* *} P<0.001$. The significance marked at the top of the columns refers to comparison with the $\mathrm{C}$ group.

IGF-1 reached 50, 100, and $200 \mathrm{ng} / \mathrm{mL}$, the cell viabilities were all remarkably increased compared with control $(F(4,10)=51.35$, $P<0.001$ ) (Figure 2A). Furthermore, it seemed that the cell viability was enhanced in a concentration-dependent manner when the concentration of IGF-1 was lower than $100 \mathrm{ng} / \mathrm{mL}$. Besides, the induction was saturated with the addition of IGF1 up to $100 \mathrm{ng} / \mathrm{mL}$; thus, the concentration of IGF-1 used in the following experiments was $100 \mathrm{ng} / \mathrm{mL}$.

MTT assays demonstrated that the cell viability of hypoxic NSCs was notably depressed compared with control $(F(3,8)=25.65$, $P<0.001$ ) (Figure $2 B$ ). With the addition of IGF-1, the viability of hypoxic NSCs increased, resulting in no obvious difference in comparison with control. However, the addition of both PPP and IGF-1 markedly reduced the viability of NSCs in comparison with control $(F(3,8)=25.65, P<0.001)$. Hence, we reached the conclusion that IGF-1 promoted cell viability of hypoxic NSCs.

\section{IGF-1 suppressed cell apoptosis of hypoxic NSCs}

The apoptosis assays of the four groups of NSCs in Figure $3 \mathrm{~A}$ and $3 \mathrm{~B}$ suggested that the apoptotic rates of treated NSCs were all remarkably improved compared with control $(F(3,8)=101.9$, $P<0.001)$. Further comparison showed that IGF-1 suppressed hypoxia-induced apoptosis in NSCS $(F(3,8)=101.9, P<0.001)$, while PPP weakened the suppression that came from IGF-1 $(F$ $(3,8)=101.9, P<0.001)$. We therefore concluded that IGF-1 suppressed cell apoptosis of hypoxic NSCs.

The protein expression levels of apoptosis-associated factors were further investigated by Western blot analysis. Figure $3 C$ and 3D show that the expression levels of Bax, cytochrome $c$, and activated caspase- 3 were all up-regulated in hypoxic NSCs compared with control $(F(3,8)=42.41, P<0.001 ; F(3,8)=19.36$, $P<0.001 ; \mathrm{F}(3,8)=67.49, P<0.001)$. When the IGF-1 was added to the culture, the expression levels of these three proteins were all down-regulated, resulting in no significant difference between cells in the $\mathrm{HI}$ group and cells in the $\mathrm{C}$ group. When IGF-1 and PPP were both added to the culture, the expression levels of these three proteins were all up-regulated and were significantly different in comparison with control $(F(3,8)=42.41$, $P<0.001 ; \mathrm{F}(3,8)=19.36, P<0.01 ; \mathrm{F}(3,8)=67.49, P<0.001)$. In terms of $\mathrm{BCl}-2$, the expression level was just the opposite of that of the three proteins described above. All these results implied that IGF-1 suppressed the cell apoptosis of hypoxic NSCs.

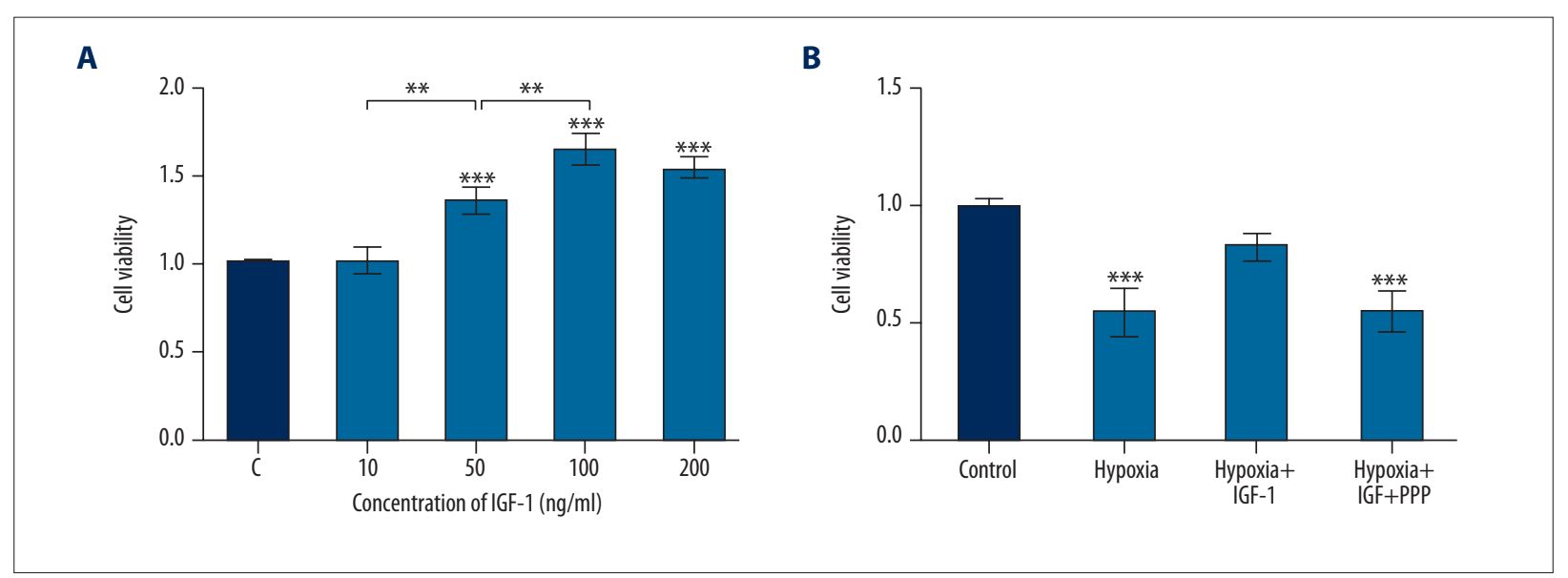

Figure 2. Insulin growth factor 1 (IGF-1) improved cell viability in hypoxic neural stem cells (NSCs). Cell viability was measured by 3-(4,5-dimethylthiazol-2-yl)-2, 5-diphenyltetrazolium bromide (MTT) assay. (A) Hypoxic NSCs were cultured under different concentrations of IGF-1. (B) NSCs were divided into four groups: the Control, Hypoxia, Hypoxia+IGF-1, and Hypoxia+IGF-1+PPP groups. Data presented are the mean \pm SD of three independent experiments. ${ }^{* *} P<0.01 ;{ }^{* *} P<0.001$. The significance marked at the top of the columns refers to comparison with the $\mathrm{C}$ group. 
A
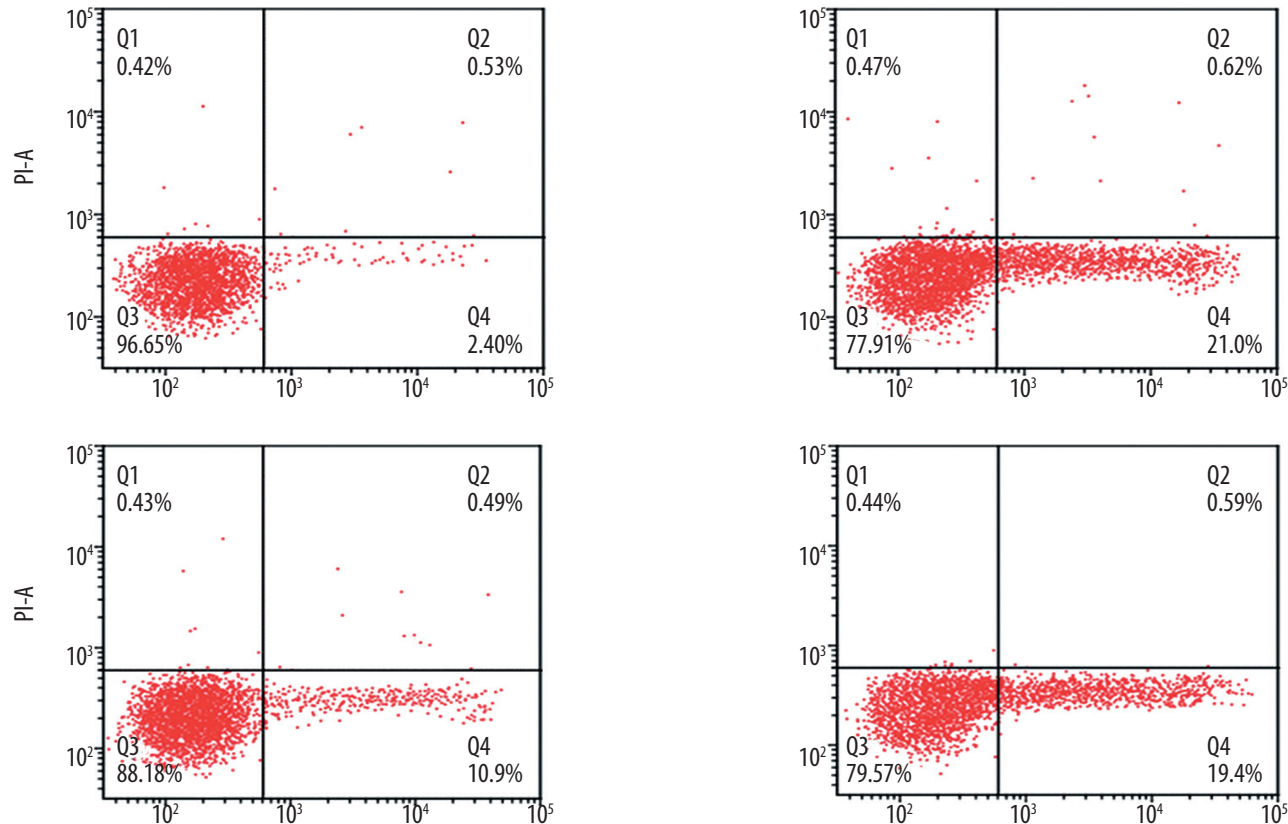

B

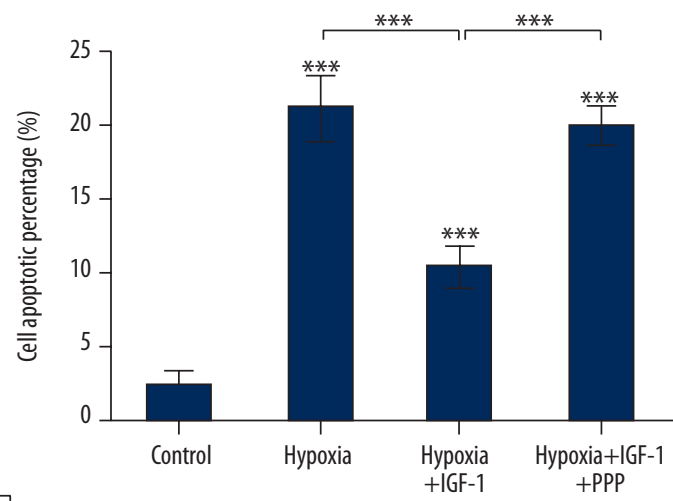

C

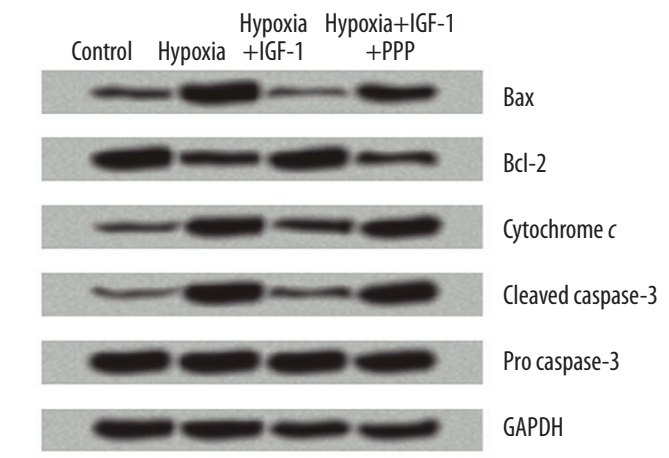

D

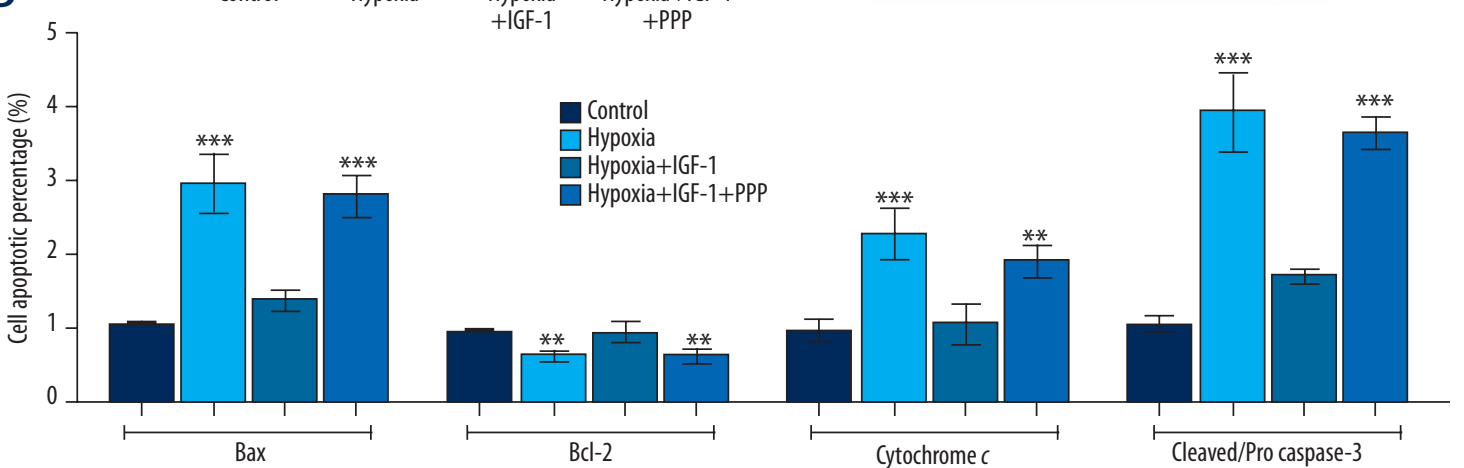

Figure 3. Insulin growth factor 1 (IGF-1) suppressed cell apoptosis induced by hypoxia in neural stem cells (NSCs). NSCs were divided into four groups: the Control, Hypoxia, Hypoxia+IGF-1, and Hypoxia+IGF-1+PPP groups. (A, B) NSCs were double stained by Annexin V-FITC and propidium iodide (PI), and then apoptotic cells were analyzed by flow cytometry. (C) The protein expression levels of apoptosis-associated factors were assayed by Western blot analysis. (D) The band intensity was assessed by Image Lab ${ }^{\mathrm{TM}}$ Software. The expression levels were all normalized by GAPDH. The expression level of activated caspase- 3 was expressed as the relative intensity of cleaved/pro caspase- 3 . Data presented are the mean \pm SD of three independent experiments. ${ }^{* *} P<0.01$; ${ }^{* \star *} P<0.001$. The significance marked at the top of the columns refers to comparison with the control group. 


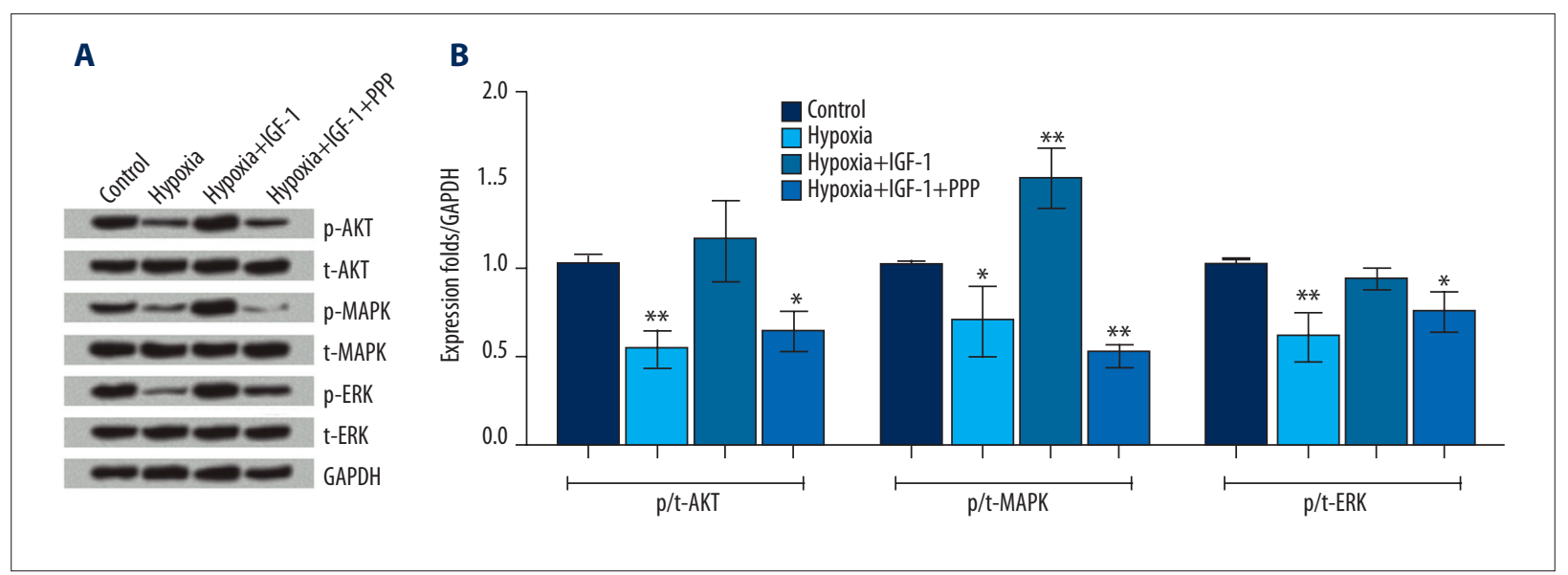

Figure 4. Insulin growth factor 1 (IGF-1) activated the phosphatidylinositol-3-kinase (PI3K)/AKT and the mitogen-activated protein kinase/extracellular signal-regulated kinase (MAPK/ERK) signaling pathways. Neural stem cells (NSCs) were divided into four groups: the Control, Hypoxia, Hypoxia+IGF-1, and Hypoxia+IGF-1+PPP groups. (A) Protein expression levels of kinases were assayed by Western blot analysis. (B) The band intensity was assessed by Image Lab ${ }^{\text {TM }}$ Software. The phosphorylation rate was expressed as the relative intensity of phosphorylated kinases/kinases, and the final results were normalized by GAPDH. Data presented are the mean \pm SD of three independent experiments. ${ }^{*} P<0.05 ;{ }^{* *} P<0.01$. The significance marked at the top of the columns refers to comparison with the control group.

\section{IGF-1 activated PI3K/AKT and MAPK/ERK pathways}

IGF-1 was added alone or in combination with its inhibitor PPP to hypoxic NSCs in order to explore the potential interactions between IGF-1 and apoptosis by Western blot analyses. Figure 4A and 4B show that the phosphorylation levels of AKT, MAPK, and ERK were all decreased in hypoxic NSCs compared with control $(\mathrm{F}(3,8)=12.95, P<0.01 ; \mathrm{F}(3,8)=33.16, P<0.05 ; \mathrm{F}$ $(3,8)=10.14, P<0.01)$. When the IGF-1 was added, the phosphorylation levels of AKT, MAPK, and ERK were all markedly improved, resulting in no significant difference or a marked increase in comparison with control $(F(3,8)=33.16, P<0.01)$. When the PPP was added, the up-regulation of p-AKT, p-MAPK, and $p$-ERK was eliminated, resulting in significant decreases in the phosphorylation levels of these three kinases in comparison with control $(F(3,8)=12.95, P<0.05 ; F(3,8)=33.16, P<0.01$; $F(3,8)=10.14, P<0.05)$. These results allowed us to reach a conclusion that IGF-1 inhibited hypoxia-induced apoptosis through activation of the PI3K/AKT and MAPK/ERK pathways.

\section{Discussion}

Acute mortality and chronic neurological disability are the main effects of HIE, which occurs in the fetus and newborn infant [25]. Although the use of hypothermia as a treatment strategy is widespread [26], the mortality and neurological disability from HIE have not obviously declined. Accelerating demands for effective HIE therapy are driving a sharp increase in the number of investigations. In this study, we constructed a hypoxia model to explore whether IGF-1 can protect NSCs from apoptosis.
We found that IGF-1 significantly increased the cell viability in hypoxic NSCs, while the increase was eliminated when PPP was added. After that, we demonstrated that IGF-1 remarkably suppressed cell apoptosis in hypoxic NSCs, while the suppression declined when PPP was added. Furthermore, we explored the protein expression levels of apoptosis-associated factors and key kinases involved in the PI3K/AKT and MAPK/AKT pathways. The expression levels of Bax, cytochrome $c$, and activated caspase-3 were obviously down-regulated, while the expression level of $\mathrm{Bcl}-2$ was prominently up-regulated by addition of IGF-1 in hypoxic NSCs. The effects of IGF-1 were reversed by addition of PPP. The studies on signaling pathway showed that IGF-1 activated the PI3K/AKT and MAPK/AKT pathways.

In general, hypoxia is considered to cause neural tissue damage in vivo and is involved in cell death, although a recent study indicated that hypoxia could facilitate proliferation of myoblast stem cells [10]. In our report, cell viability was also enhanced when the duration of hypoxia was $2 \mathrm{~h}$, which was in line with the literature cited above. In addition, we found that cell viability was markedly decreased when NSCs were exposed to hypoxia for $4 \mathrm{~h}$ or more than $4 \mathrm{~h}$. Moreover, the decreased cell viability of hypoxic NSCs could be reversed by IGF-1. The ability of IGF-1 to accelerate cell viability was also found in the previous literature. A report declared that IGF-1 promoted myoblast proliferation [27]. Furthermore, Yan et al. demonstrated that IGF-1 promoted cell proliferation of human periodontal ligament stem cells [28].

Previous research claimed that a long period of hypoxia might promote NSC apoptosis [29]. In this study, apoptosis assays 
implied that exposure to hypoxia for $6 \mathrm{~h}$ significantly promoted cell apoptosis. A majority of investigations revealed that the most common way to cell apoptosis was the mitochondrial pathway. Signals of cellular stress transferred to mitochondria resulted in mitochondrial outer membrane permeabilization (MOMP). The process of MOMP is orchestrated by the $\mathrm{Bcl}-2$ family protein-protein interactions [30]. Both pro-apoptotic and anti-apoptotic proteins exist in the $\mathrm{Bcl}-2$ protein family. Bax is one of the pro-apoptotic proteins that mediate MOMP, while $\mathrm{Bcl}-2$ is one of the anti-apoptotic proteins that inhibit MOMP [31]. The process of MOMP triggers the release of cytochrome $c$. Subsequently, the caspase pathway is activated, which gives rise to activation of downstream orchestrations of apoptotic cellular dismantling and clearance [32]. Caspase-3 is the main effector caspase, which exists as an inactive proenzyme and undergoes proteolytic processing to produce an active enzyme [33]. In this report, the pro-apoptotic protein Bax, cytochrome $c$, and activated caspase- 3 were all up-regulated in hypoxia NSCs, while the increase was eliminated by addition of IGF-1. Reasonably, the addition of PPP was observed to eliminate the function of IGF-1. The expression level of anti-apoptotic Bcl-2 was just opposite to that of the other three proteins. All the results suggested that IGF-1 could protect NSCs from hypoxia-induced apoptosis.

IGF-1 has been proven to bind tightly with IGF-1R. IGF-1R is activated with the binding and triggers multiple downstream

\section{Reference:}

1. Vhaouari P: Prevalence, causes, and outcome at 2 years of age of newborn encephalopathy: Population based study. Arch Dis Child Fetal Neonatal Ed, 2005; 90: 257-61

2. Van LH, de Haan TR, Offringa $M$ et al: Prognostic tests in term neonates with hypoxic-ischemic encephalopathy: A systematic review. Pediatrics, 2013; 131: 88-98

3. Chalak LF, Sánchez PJ, Adams-Huet B et al: Biomarkers for severity of neonatal hypoxic-ischemic encephalopathy and outcomes in newborns receiving hypothermia therapy. J Pediatr, 2014; 164: 468-74

4. Kurinczuk JJ, White-Koning M, Badawi N: Epidemiology of neonatal encephalopathy and hypoxic-ischaemic encephalopathy. Early Hum Dev, 2010; 86: 329-38

5. Higgins RD, Raju T, Edwards AD et al: Hypothermia and other treatment options for neonatal encephalopathy: An executive summary of the Eunice Kennedy Shriver National Institute of Child Health and Human Development Workshop. J Pediatr, 2011; 159: 851-58.e1

6. Rink C, Khanna S: Significance of brain tissue oxygenation and the arachidonic acid cascade in stroke. Antioxid Redox Signal, 2011; 14: 1889-903

7. Neubauer JA, Sunderram J: Oxygen-sensing neurons in the central nervous system. J Appl Physiol (1985), 2004; 96: 367-74

8. Molyneaux BJ, Arlotta P, Menezes JR, Macklis JD: Neuronal subtype specification in the cerebral cortex. Nat Rev Neurosci, 2007; 8: 427-37

9. Shoemaker LD, Kornblum HI: Neural Stem Cells (NSCs) and proteomics. Mol Cell Proteomics, 2016; 15: 344-54

10. Xiang L, Zhu L, Chen X, Ming F: Effects of hypoxia on proliferation and differentiation of myoblasts. Med Hypotheses, 2007; 69: 629-36

11. Studer L, Csete M, Lee SH et al: Enhanced proliferation, survival, and dopaminergic differentiation of CNS precursors in lowered oxygen. J Neurosc, 2000; 20: 7377-83 signaling pathways. Among them, the PI3K/AKT and MAPK/ERK pathways are relevant to cell proliferation and survival [34]. IGF-1R affects the MAPK/ERK pathway via phosphorylating a sequence of protein kinases and then controls apoptosis. A report implied that inhibition of the MAPK/ERK pathway triggered apoptosis [35]. As for the PI3K/AKT pathway, activated IGF-1R activates PI3K and then the downstream phospholipids are produced. AKT is activated by phospholipids and then interacts with Bax and Bcl-2 [36]. These apoptosis-associated proteins thereby make a great difference regarding apoptosis. In agreement, we provided evidence that IGF-1 remarkably up-regulated the phosphorylation of AKT, MAPK, and ERK in hypoxic NSCs and that the up-regulation was eliminated by PPP. These results, as well as the reports described above, support the conclusion that IGF-1 protected NSCs from hypoxia-induced apoptosis through activating the MAPK/ERK and PI3K/AKT pathways.

\section{Conclusions}

We speculate for the first time that IGF-1 could protect NSCS from hypoxia-induced apoptosis through activation of the PI3K/AKT and MAPK/ERK pathways. More studies still must be done to examine in detail the interactions between IGF-1R and kinases. The findings might help in further exploring the clinical therapies for HIE.

12. Zhu LL, Wu LY, Yew DT, Fan M: Effects of hypoxia on the proliferation and differentiation of NSCs. Mol Neurobiol, 2005; 47: 231-42

13. Ezashi T, Das $P$, Roberts RM: Low $O 2$ tensions and the prevention of differentiation of hES cells. Proc Natl Acad Sci USA, 2005; 102: 4783-88

14. Bath C, Yang S, Muttuvelu D et al: Hypoxia is a key regulator of limbal epithelial stem cell growth and differentiation. Stem Cell Res, 2013; 10: 349-60

15. Rinderknecht $E$, Humbel RE: The amino acid sequence of human insulin-like growth factor I and its structural homology with proinsulin. J Biol Chem, 1978; 253: 2769-76

16. Pucilowska JB, Davenport ML, Kabir I et al: The effect of dietary protein supplementation on insulin-like growth factors (IGFs) and IGF-binding proteins in children with shigellosis. J Clin Endocrinol Metab, 1993; 77: 1516-21

17. Ma J, Sawai H, Matsuo $Y$ et al: IGF-1 mediates PTEN suppression and enhances cell invasion and proliferation via activation of the IGF-1/PI3K/Akt signaling pathway in pancreatic cancer cells. J Surg Res, 2010; 160: 90-101

18. Supeno NE, Pati S, Hadi RA et al: IGF-1 acts as controlling switch for longterm proliferation and maintenance of EGF/FGF-responsive striatal neural stem cells. Int J Med Sci, 2012; 10: 522-31

19. Huat TJ, Khan AA, Pati S et al: IGF-1 enhances cell proliferation and survival during early differentiation of mesenchymal stem cells to neural progenitor-like cells. BMC Neurosc, 2014; 15: 1-13

20. Rubovitch V, Edut $S$, Sarfstein $R$ et al: The intricate involvement of the Insulin-like growth factor receptor signaling in mild traumatic brain injury in mice. Neurobiol Dis, 2010; 38: 299-303

21. Girnita A, All-Ericsson C, Economou MA et al: The insulin-like growth fac tor-I receptor inhibitor picropodophyllin causes tumor regression and attenuates mechanisms involved in invasion of uveal melanoma cells. Clin Cancer Res, 2006; 12(4): 1383-91 
22. Vasilcanu R, Vasilcanu D, Rosengren L et al: Picropodophyllin induces downregulation of the insulin-like growth factor 1 receptor: Potential mechanistic involvement of Mdm2 and beta-arrestin1. Oncogene, 2008; 27: 1629-38

23. Zhang $X$, Zhang L, Cheng $X$ et al: IGF-1 promotes Brn-4 expression and neuronal differentiation of neural stem cells via the PI3K/Akt pathway. PLoS One, 2014; 9: e113801

24. Haiyan H, Rensong Y, Guoqin J et al: Effect of Astragaloside IV on neural stem cell transplantation in Alzheimer's disease rat models. Evid Based Complement Alternat Med, 2016; 2016: 3106980

25. Dai Y, Li W, Zhong M et al: Preconditioning and post-treatment with cobalt chloride in rat model of perinatal hypoxic-ischemic encephalopathy. Brain Dev, 2014; 36: 228-40

26. Merchant NM, Azzopardi DV, Edwards AD: Neonatal hypoxic ischaemic encephalopathy: Current and future treatment options. Expert Opinion on Orphan Drugs, 2015; 3: 357-77

27. Yu M, Wang H, Xu Y et al: Insulin-like growth factor-1 (IGF-1) promotes myoblast proliferation and skeletal muscle growth of embryonic chickens via the PI3K/Akt signalling pathway. Cell Biology International, 2015; 39: 910-22

28. Yan Y, Mu J, Fan Z et al: Insulin-like growth factor 1 enhances the proliferation and osteogenic differentiation of human periodontal ligament stem cells via ERK and JNK MAPK pathways. Histochem Cell Biol, 2012; 137: 513-25
29. Yuan L, Guan Y, Du H, Ma D: The Effects of hypoxia on proliferation, differentiation and apoptosis of cultured NSCs from brain cortex of embryonic rats in vitro. Chinese Journal of Neuroanatomy, 2008; 24: 343-48

30. Chipuk JE, Moldoveanu T, Llambi F et al: The BCL-2 family reunion. Mol Cell, 2010; 37: 299-310

31. Llambi F, Moldoveanu T, Tait SG et al: A unified model of mammalian BCL-2 protein family interactions at the mitochondria. Mol Cell, 2011; 44: 517-31

32. Li Y, Zhang S, Geng JX, Hu XY: Curcumin inhibits human non-small cell lung cancer A549 cell proliferation through regulation of $\mathrm{Bcl}-2 / \mathrm{Bax}$ and cytochrome C. Asian Pac J Cancer Prev, 2013; 14(8): 4599-602

33. Loison $\mathrm{F}$, Zhu H, Karatepe $\mathrm{K}$ et al: Proteinase 3 - dependent caspase-3 cleavage modulates neutrophil death and inflammation. J Clin Invest, 2014; 124: 4445-58

34. Valenciano A, Moreno $M$, Lloret $M$, Lara PC: Role of IGF-1 receptor in radiation response. Transl Oncol, 2012; 5: 1-9

35. Papademetrio DL, Lompardía SL, Simunovich T et al: Inhibition of survival pathways MAPK and NF- $\mathrm{KB}$ triggers apoptosis in pancreatic ductal adenocarcinoma cells via suppression of autophagy. Targeted Oncology, 2015; 11: 1-13

36. Sell C, Baserga R, Rubin R: Insulin-like growth factor I (IGF-I) and the IGF-I receptor prevent etoposide-induced apoptosis. Cancer Res, 1995; 55: 303-6 